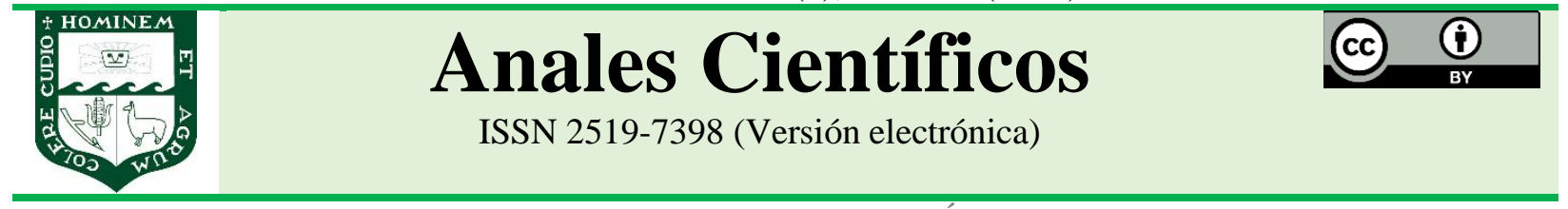

ARTÍCULO ORIGINAL - RESEARCH ARTICLE

http://dx.doi.org/10.21704/ac.v81i2.1648

\title{
EFECTO DEL FOTOPERIODO Y EL USO DE UN NUTRIENTE FOLIAR COMERCIAL EN EL CRECIMIENTO (BIOMASA) DE Pyropia sp. CHI “COCHAYUYO" BAJO CONDICIONES CONTROLADAS DE CULTIVO
}

\section{Effect of the photoperiod and the use of a commercial foliar nutrient on the growth (Biomass) of Pyropia sp. CHI "Cochayuyo" under controlled cultivation conditions}

\author{
Samuel Arbaiza ${ }^{1,2}$; Max Castañeda ${ }^{2}$; Orlando Advíncula ${ }^{3 * D}$; Víctor Meza ${ }^{1}$ \\ ${ }^{1}$ Facultad de Ciencias, Universidad Nacional Agraria La Molina, 15024, Lima, Perú. \\ ${ }^{2}$ Universidad Científica del Sur, Lima, Perú. \\ ${ }^{3}$ Universidad San Ignacio de Loyola, Lima, Perú. \\ *E-mail: orlandoad@gmail.com
}

Recibido: 22/07/2019; Aceptado: 15/12/2020; Publicado: 30/12/2020

\begin{abstract}
In order to determine the effect of photoperiod and the addition of commercial foliar nutrient at different concentrations in the biomass increasing of Pyropia sp. CHI, gametophytic individuals were collected in Santa Elena beach, Paracas - Pisco and transferred to Applied Biology Laboratory of Universidad Nacional Agraria La Molina. The thalli were divided into groups of 1,0 $\pm 0,02 \mathrm{~g}$ of initial biomass and placed in flasks with enriched sea water with three different concentrations of Bayfolan ${ }^{\circledR}$ commercial foliar nutrient $(0,1 \mathrm{ml} / \mathrm{L} ; 0,05 \mathrm{~m} / \mathrm{L}$; and 0,025 $\mathrm{ml} / \mathrm{L})$ and a control treatment $(0 \mathrm{ml} / \mathrm{L})$ against two photoperiod conditions: long day (DL) (L/O = 18:6) and short day $(\mathrm{DC})(\mathrm{L} / \mathrm{O}=6: 18)$. Biomass (wet weight) and growth rate $(\mathrm{g} / \mathrm{week})$ were evaluated weekly during six weeks. The results have demonstrated the effect of nutrient addition in biomass increase of Pyropia sp. CHI where all treatments with added nutrient, for both DC and DL, obtained significantly higher growth rates $(\mathrm{p}<0,05)$ than treatment without nutrient addition $(0 \mathrm{ml} / \mathrm{L})$. Likewise, a higher biomass was obtained for DL condition with 1,53 $\mathrm{g}(0 \mathrm{~m} / \mathrm{L}) ; 2,98 \mathrm{~g}(0,025 \mathrm{ml} / \mathrm{L}) ; 3,28 \mathrm{~g}(0,05 \mathrm{ml} / \mathrm{L})$ and $3,57 \mathrm{~g}(0,1 \mathrm{ml} / \mathrm{L})$ unlike that obtained in DC condition with $1,42 \mathrm{~g}(0 \mathrm{ml} / \mathrm{L}) ; 2,79 \mathrm{~g}(0,025 \mathrm{ml} / \mathrm{L}) ; 2,66 \mathrm{~g}(0,05 \mathrm{ml} / \mathrm{L})$ and $2,65 \mathrm{~g}(0,1 \mathrm{ml} / \mathrm{L})(\mathrm{p}<0,05)$.
\end{abstract}

Keywords: Cochayuyo; Pyropia sp. CHI; Bayfolan®; photoperiod; culture

\section{RESUMEN}

Con la finalidad de determinar el efecto del fotoperiodo y la adición de un nutriente comercial foliar a diferentes concentraciones en el aumento de biomasa de Pyropia sp. CHI, individuos gametofíticos fueron colectados en playa Santa Elena, Paracas - Pisco y trasladados al Laboratorio de Biología Aplicada de la Universidad Nacional Agraria La Molina. Los talos fueron divididos en grupos de 1,0 $\pm 0,02 \mathrm{~g}$ de biomasa inicial y puestos en matraces con agua de mar enriquecida a tres diferentes concentraciones de nutriente foliar comercial Bayfolan ${ }^{\circledR}(0,1 \mathrm{ml} / \mathrm{L}, 0,05 \mathrm{~m} / \mathrm{L}$ y $0,025 \mathrm{ml} / \mathrm{L})$ y un tratamiento control $(0 \mathrm{ml} / \mathrm{L})$ frente a dos condiciones de fotoperiodo: día largo $(\mathrm{DL})(\mathrm{L} / \mathrm{O}=18: 6)$ y día corto $(\mathrm{DC})(\mathrm{L} / \mathrm{O}=6: 18$. Se evaluó semanalmente la biomasa (peso húmedo) y la tasa de crecimiento 
(g/semana) durante seis semanas. Los resultados han demostrado el efecto de la adición de nutriente en el aumento de biomasa de Pyropia sp. CHI en donde todos los tratamientos con adición de nutriente, tanto para DC como DL, obtuvieron tasas de crecimiento significativamente mayores $(p<0,05)$ que el tratamiento sin adición de nutriente $(0 \mathrm{ml} / \mathrm{L})$. Así mismo, se obtuvo una mayor biomasa para la condición de DL con 1,53 g $(0 \mathrm{ml} / \mathrm{L}) ; 2,98 \mathrm{~g}(0,025$ $\mathrm{ml} / \mathrm{L}) ; 3,28 \mathrm{~g}(0,05 \mathrm{ml} / \mathrm{L})$ y $3,57 \mathrm{~g}(0,1 \mathrm{ml} / \mathrm{L})$ a diferencia de lo obtenido en la condición de DC con 1,42 g $(0 \mathrm{ml} / \mathrm{L})$, $2,79 \mathrm{~g}(0.025 \mathrm{ml} / \mathrm{L}) ; 2,66 \mathrm{~g}(0,05 \mathrm{ml} / \mathrm{L})$ y $2,65 \mathrm{~g}(0,1 \mathrm{ml} / \mathrm{L})(p<0,05)$.

Palabras clave: Cochayuyo; Pyropia sp. CHI; Bayfolan®; fotoperiodo; cultivo

Forma de citar el artículo (Formato APA):

Arbaiza, S., Castañeda, M., Advíncula, O. \& Meza, V. (2020). Efecto del fotoperiodo y el uso de un nutriente foliar comercial en el crecimiento (Biomasa) de Pyropia sp. CHI "Cochayuyo" bajo condiciones controladas de cultivo. Anales Científicos. 81(2), 347-354. http://dx.doi.org/10.21704/ac.v81i2.1648

Autor de correspondencia (*): Orlando Advíncula. Email: orlandoad@gmail.com

(c) Los autores. Publicado por la Universidad Nacional Agraria La Molina.

This is an open access article under the CC BY

\section{INTRODUCCIÓN}

A nivel mundial las tres algas marinas de mayor producción acuícola para el consumo humano directo son Laminaria japónica (kombu), Undaria pinnatifida (wakame) y Porphyra spp. (nori); siendo el género Porphyra/Pyropia el que presenta la mayor producción de la maricultura japonesa y un mayor valor económico a comparación del kombu y el wakame (McHugh, 2002; FAO, 2016). Dada su importancia comercial, la sistemática de Bangiales foliosas está presentando cambios significativos debido al uso de herramientas moleculares y ello ha conllevado la inclusión de nuevos géneros como Pyropia, el cual no se encontraba reportado para Perú. Recientemente, se determinaron para Perú tres especies de Porphyra y cuatro especies de Pyropia (Marquez, 2019) siendo la más abundante para la costa central la especie Pyropia sp. CHI.

El cultivo y procesamiento de Porphyra/Pyropia es una de las industrias acuícolas más importantes de las costas asiáticas, principalmente en Japón, China y Corea; considerada como el alga más valiosa de la maricultura mundial. Debido a su gran importancia económica y beneficios para la salud, el cultivo de Porphyra spp. se ha extendido a países de Europa, América, África y Oceanía (Sahoo et al., 2002; Chen \& Xu, 2005; Sahoo $\&$ Yarish, 2005).

En el Perú, Porphyra spp. (Porphyra y/o Pyropia) conocido comúnmente con el nombre de "cochayuyo" es consumido tradicionalmente en estado fresco y/o seco en diversos platos de la gastronomía peruana (Delgado, 2004; Ugás, 2014; Noriega, 2011), sin embargo, a pesar de su importancia no se ha podido desarrollar el cultivo de esta especie.

Uno de los factores más importantes para desarrollar el cultivo de esta especie a escala comercial, es determinar una fuente de nutrientes alternativa a los medios de cultivo preparados a partir de sales inorgánicas de grado analítico los cuales pueden ser restrictivos debido a su alto costo (Provasoli, Von Stoch). Diversos trabajos han sugerido el uso de fertilizantes agrícolas como alternativa de bajo costo al uso de sales inorgánicas de grado analítico para el cultivo masivo (Edding, 1995; Pacheco-Ruíz et al., 2004; Romo et al., 2005; Israel et al., 2006; Bulboa et al., 2013). Así mismo, el nutriente comercial Bayfolan $^{\circledR}$ es utilizado ampliamente en cultivos locales de microalgas y otras algas como Chondracanthus chamissoi, sin embargo, no se ha evaluado su concentración óptima para el crecimiento de Pyropia sp. CHI en las condiciones de Perú.

Por otro lado, diversos autores han determinado el efecto del fotoperiodo (horas de luz y oscuridad) en el crecimiento y desarrollo de las algas (Ávila et al;1986,. Salinas \& Valdés, 1993; Garza-Sánchez et al., 2000; Bulboa \& Macchiavello, 2001; Buschmann et al., 2004; Ávila et al., 2011; Redmond et al., 2014), y sus magnitudes dependerán de los objetivos del cultivo y las exigencias del organismo cultivado (Redmond et al., 2014). Así, por ejemplo, se puede realizar un cultivo con fotoperiodo de días largos (verano): 16:8 o 14:10 (Luz/Oscuridad) para fomentar el crecimiento y desarrollo de las algas (Oliveira et al., 1995). Sin embargo, Pyropia sp. CHI. es una especie muy estacional cuya fase foliar macroscópica (gametofítica) 
crece en el medio natural en estaciones de días cortos: desde mediados de otoño hasta finales de invierno e inicios de la primavera. Debido a ello, existe una incertidumbre en identificar las condiciones óptimas de fotoperiodo para el cultivo comercial en laboratorio de Pyropia sp. CHI.

Por lo tanto, el objetivo de la presente investigación fue determinar la condición de fotoperiodo y nutriente comercial Bayfolan ${ }^{\circledR}$ óptima para el aumento de biomasa en un cultivo de Pyropia sp. CHI en condiciones semi-controladas de laboratorio.

\section{MATERIALES Y MÉTODOS}

Con el objetivo de identificar la condición óptima de fotoperiodo y nutriente comercial Bayfolan ${ }^{\circledR}$ para el aumento de biomasa en el cultivo de Pyropia sp. CHI, individuos gametofíticos de 4 a $5 \mathrm{~cm}$. de Pyropia sp. CHI fueron colectados en playa Santa Elena, Paracas Pisco $\left(13^{\circ} 55^{\prime} \mathrm{S} 76^{\circ} 19^{\prime} \mathrm{W}\right)$ y luego trasladados en contenedores térmicos aislados a las instalaciones del Laboratorio de Biología Aplicada de la Universidad Nacional Agraria La Molina. Una vez en el laboratorio, los talos fueron lavados con agua destilada y agua de mar esterilizada para eliminar epífitos e impurezas. Posteriormente, se colocó $1,0 \pm 0,02 \mathrm{~g}$ (peso fresco) de material vegetal en matraces con 1 litro de agua de mar filtrada y esterilizada (tratamiento UV) para dar inicio al procedimiento experimental.

Se evaluaron 8 tratamientos los cuales estaban compuestos de dos factores: fotoperiodo (día largo (DL) $=18: 6$ y día corto $(\mathrm{DC})=6: 18)$ y concentración de nutriente Bayfolan ${ }^{\circledR}(0,1 \mathrm{ml} / \mathrm{L}, 0,05 \mathrm{~m} / \mathrm{L}$, y $0,025 \mathrm{ml} / \mathrm{L}$ y $0 \mathrm{ml} / \mathrm{L}$ ). Cada tratamiento tuvo 3 repeticiones (totalizando 24 unidades experimentales). Así mismo, los sistemas de cultivo fueron mantenidos con agua de mar filtrada y esterilizada a una temperatura constante de $15^{\circ} \mathrm{C}$ con una tasa de recambio semanal del $100 \%$ y un sistema de aireación permanente. La intensidad lumínica se mantuvo constante a 3300 LUX. Así mismo, con cada recambio de medio de cultivo, se agregó la concentración de nutriente determinada, se evaluó la biomasa (peso húmedo), utilizando una balanza analítica $(0,001 \mathrm{~g}$. de precisión), y se calculó la tasa de crecimiento según la formulada empleada por Bulboa (2006): TC $(\% /$ día $)=\left\{\left[(\mathrm{Tf} / \mathrm{To})^{1 / t}\right]-1\right\} .100$, donde
"Tf" es la talla final, "To", la talla inicial y "t" el tiempo de cultivo. El tiempo de análisis experimental tuvo una duración de 6 semanas.

\section{Análisis estadístico}

Para analizar las diferencias de biomasa y tasa de crecimiento respecto a los tratamientos y al tiempo de evaluación (semanas) se utilizaron modelos lineales generales y mixtos para considerar una posible heterogeneidad de varianza durante el período analizado (Di Rienzo et al., 2017), analizándolo como un experimento factorial. Luego se seleccionó el modelo más apropiado utilizando los valores del criterio de Akaike (AIC), Schwarz (BIC) y la prueba de máxima verosimilitud (LRT). Para todas las variables medidas se analizó la normalidad de los errores usando la prueba de Shapiro-Francia (Shapiro \& Francia, 1972). Para analizar la homogeneidad de varianzas, se usó un gráfico de dispersión de residuos versus valores predichos (Di Rienzo et al., 2008). De encontrarse diferencias significativas entre los tratamientos, se aplicó la prueba LSD de Fisher $(\alpha=5 \%)$.

\section{RESULTADOS Y DISCUSIÓN}

Uno de los factores más importantes para el crecimiento y desarrollo de las algas es la disponibilidad de luz (fotoperiodo) condición que afecta directamente a la productividad de cualquier vegetal (organismo fotosintético) y por otro lado la disponibilidad de nutrientes, lo cual es importante dado que cualquier organismo vegetal necesita determinadas concentraciones de macro y micronutrientes esenciales para su metabolismo (Lobban \& Harrison, 1994). Se ha demostrado que la baja disponibilidad de nutrientes (en especial nitrógeno) limita el crecimiento de las macroalgas (Lobban \& Harrison, 1994); por lo cual, se suele incorporar estos elementos a los cultivos masivos de macro y micro algas con el objetivo de obtener cultivos exitosos.

Los resultados experimentales han evidenciado el efecto de la adición de nutriente en la producción de biomasa de Pyropia sp. CHI, donde, en primer lugar, todos los tratamientos con adición de nutriente fueron significativamente mayores $(p<0,05)$ que el tratamiento control (sin nutriente) y ello se dio en ambas condiciones de fotoperiodo (DL y DC) (Figura $1 \mathrm{y}$ 
Figura 2), evidenciando en ambos fotoperiodos la interacción tratamiento y semana, la cual fue significativa $(p<0,05)$. Sin embargo, respecto al fotoperiodo, se obtuvo una mayor biomasa en la condición de días largos (DL) con 1,53 g. (0 ml/L), 2,98 g. $(0,025 \mathrm{ml} / \mathrm{L}), 3,28 \mathrm{~g}(0,05 \mathrm{ml} / \mathrm{L})$ y $3,57 \mathrm{~g}(0,1 \mathrm{ml} / \mathrm{L})$ a diferencia de lo obtenido en la condición de días cortos (DC) con 1,42 g (0 ml/L); 2,79 g (0,025 ml/L); $2,66 \mathrm{~g}(0,05 \mathrm{ml} / \mathrm{L})$ y $2,65 \mathrm{~g}(0,1 \mathrm{ml} / \mathrm{L})(p<0,05)$ (Figura 1 y Figura 2).

Estos mismos resultados se han podido evidenciar en los valores de la tasa de crecimiento (TC) donde todos los tratamientos con adición de nutriente, tanto en la condición de día largo (DL) y día corto (DC) fueron significativamente mayores que el tratamiento sin adición de nutriente $(0 \mathrm{ml} / \mathrm{L})(p<0,05)$ (Figura 3 y 4$)$.

Una de las formas de incorporar estos nutrientes es mediante la adición de fertilizantes agrícolas los cuales nos permiten obtener altos rendimientos, una buena calidad en plantas y un bajo costo a diferencia de los medios de cultivo elaborados con sales de grado analítico (Pacheco-Ruíz et al., 2004).

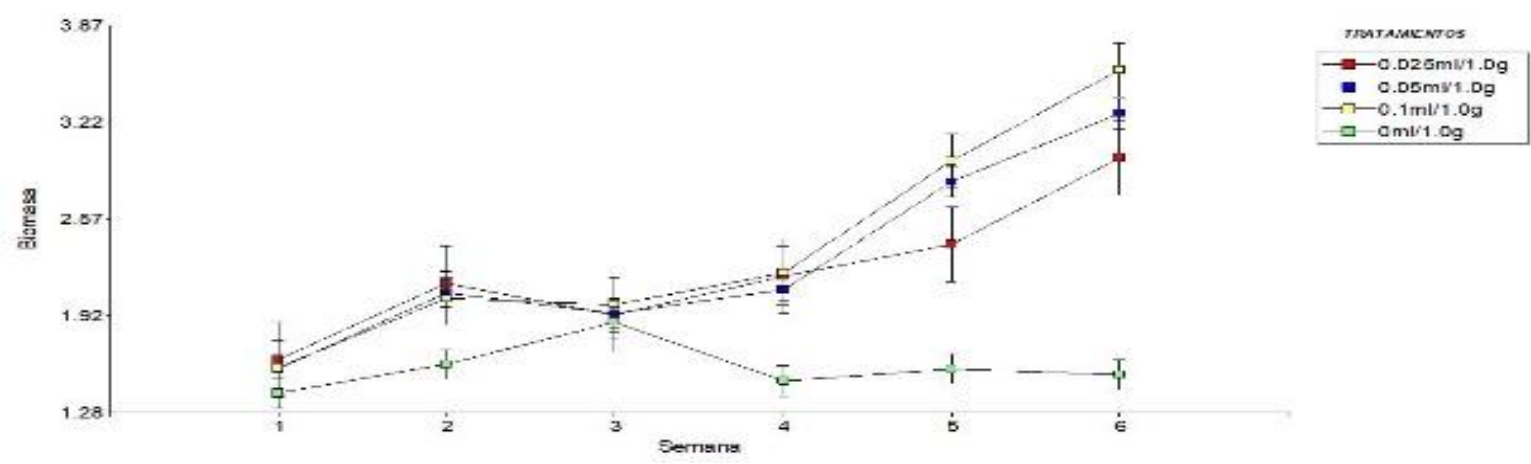

Figura 1. Aumento de biomasa de Pyropia sp. CHI a lo largo de 6 semanas de cultivo en condición de día largo (DL). Los tratamientos con adición de nutriente fueron significativamente mayores que el tratamiento control (sin nutriente) $(p<0,05)$

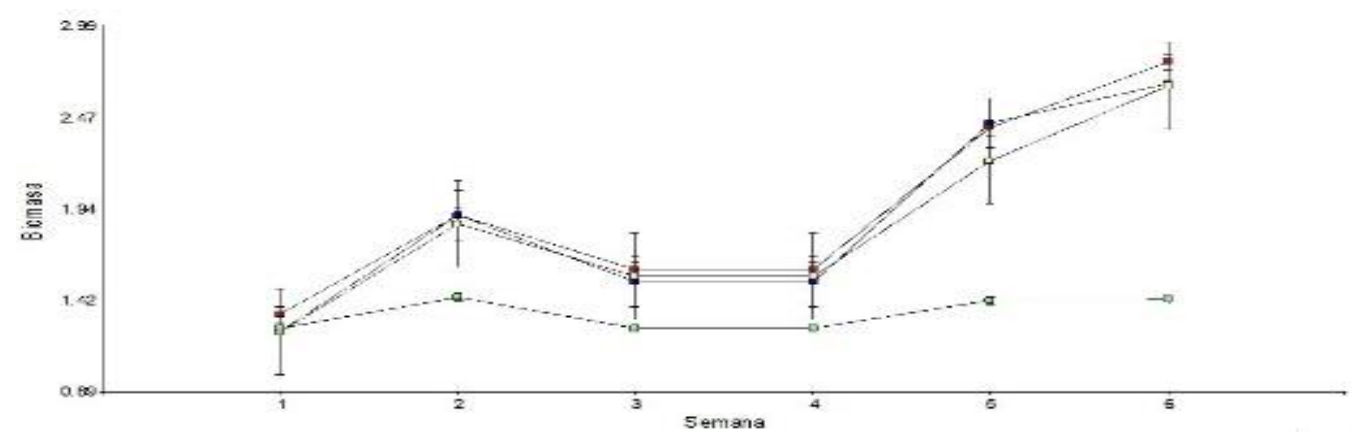

Figura 2. Aumento de biomasa de Pyropia sp. CHI a lo largo de 6 semanas de cultivo en condición de día corto (DC). Los tratamientos con adición de nutriente fueron significativamente mayores que el tratamiento control (sin nutriente) $(p<0,05)$

Diversos autores han empleado diferentes fertilizantes comerciales como medio nutritivo para cultivos de microalgas (Valenzuela-Espinoza et al., 2005; Piña et al., 2011; Morales-Ventura et al., 2012; Mercado, 2016) y macroalgas: Gracilaria chilensis (Alveal et al., 1997), Chondracanthus squarrulosus (Pacheco-Ruíz et al., 2004), Porphyra sp. (Romo et al., 2005), Sarcothalia crispata (Werlinger et al., 2008), Gigartina skottsbergii (Mansilla et al., 2008), Turbinaria conoides (Mayakun et al., 2013) y Chondracanthus chamissoi (Arbaiza, 2016; Colque, 2017; Castañeda et al., 2018). Así mismo, el nutriente comercial Bayfolan ${ }^{\circledR}$ ha sido utilizado ampliamente en cultivos masivos 
de macroalgas demostrando en muchos casos un mayor rendimiento que otros fertilizantes y medios de cultivo elaborados en base a sales de grado analítico (Mansilla et al., 2008; Colque, 2017).

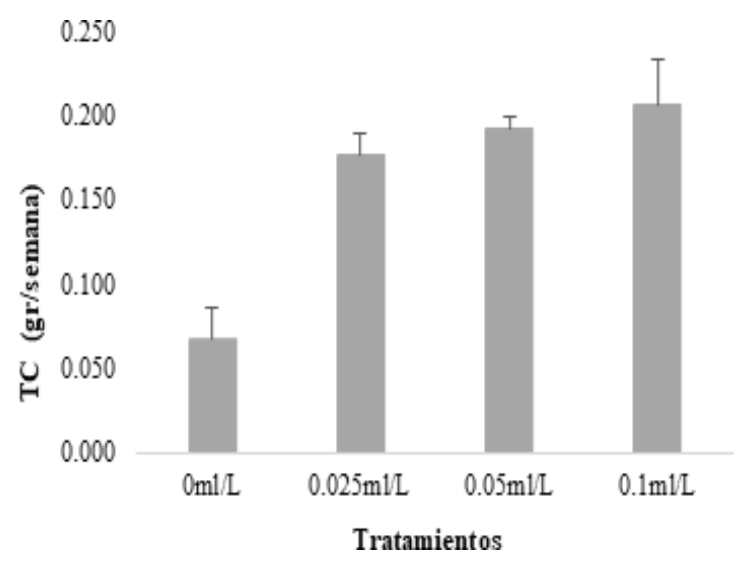

Figura 3. Tasa de crecimiento semanal de Pyropia sp. CHI en condición de día largo (DL).

Los resultados obtenidos en el presente experimento han demostrado que el aumento de biomasa de Pyropia sp. CHI ha sido ocasionado principalmente por la adición de nutriente comercial. Así mismo, el tratamiento con la concentración de $0.1 \mathrm{ml} / \mathrm{L}$ presentó valores ligeramente más altos de biomasa para la condición de DL coincidiendo con las concentraciones de Bayfolan ${ }^{\circledR}$ desarrolladas por Romo et al. (2005) para el cultivo de Pyropia sp. CHI es $0.1 \mathrm{ml} / \mathrm{L}$ y Colque (2017) para el cultivo de C. chamissoi. Sin embargo, los resultados han demostrado que con la concentración de $0.025 \mathrm{ml} / \mathrm{L}$ de Bayfolan ${ }^{\circledR}$ se puede obtener buenos resultados.

Por otro lado, Pyropia sp. CHI es una especie estacional, donde la fase foliosa (gametofítica) aparece a mediados de otoño y crece a lo largo de todo el invierno, época donde las temperaturas del agua son más bajas y el fotoperiodo se reduce (Días cortos .(Sin embargo, los resultados han demostrado que los mayores aumentos de biomasa en todos los tratamientos han ocurrido en la condición de días largos (16 horas luz, 8 horas oscuridad). Ávila et al. (1986) determinó que el crecimiento de frondas juveniles de Porphyra columbina ocurre a $15^{\circ} \mathrm{C}$ y con condiciones de fotoperiodo de día largo ( $\mathrm{L} / \mathrm{O}=$ 16:08). Así mismo, Redmond et al. (2014) señala algo similar con diversas especies del género
Porphyra. Por lo tanto, el mayor aumento de biomasa se debe a la mayor cantidad de luz disponible para la actividad fotosintética.

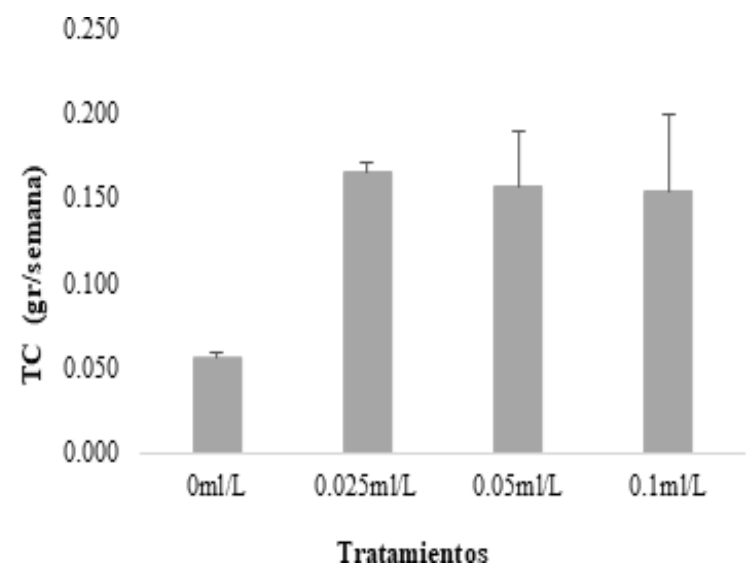

Figura 4. Tasa de crecimiento semanal de Pyropia sp. CHI en condición de día corto (DC)

\section{CONCLUSIONES}

La condición óptima de cultivo para el crecimiento de Pyropia sp. CHI en condiciones de laboratorio, es un fotoperiodo de día largo (16:8) y con adición del nutriente comercial Bayfolan ${ }^{\circledR}$ en una concentración de $0,025 \mathrm{ml} / \mathrm{L}$, la cual obtuvo biomasas ligeramente menores a las concentraciones de 0.1 y $0,05 \mathrm{ml} / \mathrm{L}$, sin embargo, al tener menor concentración de nutriente es más recomendable en temas económicos.

\section{Agradecimientos}

Los autores agradecen a INNOVATEPERU por el financiamiento brindado para el desarrollo del proyecto Contrato No 137 IAPIP-2017. Así mismo, se agradece al equipo del Laboratorio de Biología Aplicada por su apoyo incondicional y a los miembros de la Cooperativa de Pescadores Artesanales Algas Marinas de Pisco (COTRAPALMAR) y al Laboratorio de Investigación en Cultivos Marinos (LICMA) por brindar las facilidades logísticas para la colecta de material biológico. 


\section{Conflictos de intereses}

Los autores firmantes del presente trabajo de investigación declaran no tener ningún potencial conflicto de interés personal o económico con otras personas $\mathrm{u}$ organizaciones que puedan influir indebidamente con el presente manuscrito.

\section{Contribuciones de los autores}

Preparación y ejecución: SA, MC, OA, VM; Desarrollo de la metodología: SA, MC, OA, VM; Concepción y diseño: SA, MC, OA, VM; Edición del artículo: SA, MC, OA, VM; Supervisión del estudio: SA, MC, OA, VM.

\section{LITERATURA CITADA}

- Alveal, K., Romo, H., Werlinger, C., \& Oliveira, E.C. (1997). Mass cultivation of the agarproducing alga Gracilaria chilensis (Rhodophyta) from spores. Aquaculture, 148(23): 77-83.

- Arbaiza, S. (2016). Viabilidad reproductiva para el cultivo de Chondracanthus chamissoi proveniente de tres poblaciones del litoral peruano. Tesis para optar por el grado de Magister Scientiae en Acuicultura. Escuela de Post-grado. Universidad Nacional Agraria La Molina. 22 p.

- Ávila, M., Santelices, B., \& McLachlan, J. (1986). Photoperiod and temperature regulation of the life history of Porphyra columbina (Rhodophyta, Bangiales) from central Chile. Canadian Journal of Botany, 64(9):1867-1872.

- Ávila, M., Piel, M., Cáceres, J., \& Alveal, K. (2011). Cultivation of the red alga Chondracanthus chamissoi: sexual reproduction and seeding production in culture under controlled conditions. J. Appl. Phycol., 23:529536.

- Bulboa, R.C., \& Macchiavello, E. J. (2001). The effects of the light and temperature on different phases of the life cycle in the carrageenan producing alga Chondracanthus chamissoi (Rhodophyta, Gigartinales). Botanica Marina, 44: 371-374.

- Bulboa, C.C. (2006). Bases bio-tecnológicas para o cultivo de Chondracanthus chamissoi, uma alga vermelha de importância econômica da costa chilena. Tese apresentada ao Instituto de Biociências da Universidade de São Paulo, para a obtenção de Título de Doutor em Ciências, na Área de Botânica. Brasil.

- Bulboa, C., Véliz, K., Sáez, F., Sepúlveda, C., Vega, L., \& Macchiavello, J. (2013). A new method for cultivation of the carragenophyte and edible red seaweed Chondracanthus chamissoi based on secondary attachment disc: Development in outdoor tanks. Aquaculture, 410-411: 86-94.

- Buschmann, H.A., Vásquez, J.A., Osorio, P., Reyes, E., Filún, L., Hernández-Gonzáles, M.C., $\&$ Vega, A. (2004). The effect of water movement, temperature and salinity on abundance and reproductive patterns of Macrocystis spp. (Phaeophyta) at differents latitudes in Chile. Marine Biology, 145: 849862.

- Castañeda, M., Arbaiza, S., Díaz, F., Castillo, Y., Baltazar, P., \& Advíncula, O. (2018). Evaluación del fotoperiodo en el asentamiento de tetraesporas de Chondracanthus chamissoi sobre cuerdas de polipropileno en condiciones semi-controladas de laboratorio. Universidad Nacional Agraria La Molina. Anales Científicos, 79(2): 459-465.

- Colque, L.M. (2017). Evaluación del crecimiento de cultivo vegetativo de (Chondracanthus chamissoi), utilizando fertilizante comercial bayfolan y medio guillard $\mathrm{f} / 2$, en condiciones de laboratorio en el centro de acuicultura morro sama del Fondepes. Para optar el Título Profesional de Ingeniero Pesquero. Universidad Nacional Jorge Basadre Grohmann.

- Chen, J., \& Xu, P. (2005). Culture aquatic species information programme - Porphyra spp. En: Departamento de pesca y acuicultura de la FAO (en línea). Roma. Disponible en http://www.fao.org/fishery/culturedspecies/Por phyra_spp/es.

- Delgado, H.E. (2004). Plantas alimenticias del Perú. Departamento Académico de Nutrición clínica y comunitaria. Universidad Científica del Sur. Lima - Perú.

- Di Rienzo, J.A., Casanoves, F., Balzarini, M.G., Gonzalez, L., Tablada, M., \& Robledo, C.W. (2008). InfoStat, versión 2008, Grupo InfoStat, 
FCA, Universidad Nacional de Córdoba, Argentina. 115.

- Di Rienzo, J.A., Macchiavelli, R.E., \& Casanoves, F. (2017). Modelos Lineales Mixtos Aplicaciones en InfoStat. Primera edición, 3.

- Edding, M.E. (1995). Cultivo de Gracilaria en estanques. En Alveal, K.; Ferrario, M. E.; Oliveira, E. C.; Sar, E. 1995. Manual de Métodos Ficológicos. Universidad de Concepción. Chile. Pág. 577-597.

- FAO. (2016). The state of world fisheries and aquaculture: contributing to food security and nutrition for all. Roma. $200 \mathrm{p}$.

- Garza-Sánchez, F., Zertuche-Gonzáles, J.A., \& Chapman, D. J. (2000). Effect of temperature and irradiance on the release, attachment and survival of spores of Gracilaria pacifica Abbott (Rhodophyta). Botanica Marina, 43: 205-212.

- Israel, A., Levy, I., \& Friedlander, M. (2006). Experimental tank cultivation of Porphyra in Israel. Journal of Applied Phycology, 18: 235240.

- Lobban, R., \& Harrison, P. (1994). Seaweed Ecology and Physiology. Cambridge University Press. Pag.: 163-170.

- Mansilla, A., Palacios, M., Navarro, N.P., Avila, M. (2008). Growth and survival performance of the gametophyte of Gigartina skottsbergii (Rhodophyta, Gigartinales) under defined nutrient conditions in laboratory culture. Journal of Applied Phycology, 20(5): 889-896.

- Marquez, D. (2019). Delimitación de especies de los géneros Pyropia/Porphyra (Bangiales, Rhodophyta) de la costa peruana en base a marcadores moleculares. Tesis para obtener el título profesional de Biólogo. Facultad de Ciencias. Universidad Nacional Agraria La Molina, pp 30.

- Mayakun, J., Kim, J.H., Lapointe, B.E., \& Prathep, A. (2013). Effects of nutrient enrichment and herbivory on morphology, reproduction and chemical content of Turbinaria conoides (Phaeophyceae). Phycological Research, 61(4): 270-276.

- McHugh, D.J. (2002). Perspectivas para la producción de algas marinas en los países en desarrollo: la industria de algas marinas. FAO Circular de Pesca No 968. Roma. 30 p.
- Mercado, E. (2016). Cultivo de la microalga Scenedesmus Obliquus var. Dimorphus (TURPIN) para la obtención de biomasa y lípidos. Universidad Ricardo Palma. Retrieved from Disponible en http://repositorio.urp.edu.pe/handle/urp/973.

- Morales-Ventura, J., Nandini, S., Sarma, S., \& Castellanos-Páez, M. (2012). Demography of zooplankton (Anuraeopsis fissa, Brachionus rubens and Moina macrocopa) fed Chlorella vulgaris and Scenedesmus acutus cultured on different media. Revista de Biología Tropical, 60(3): 955-965.

- Noriega, C. (2011). Algas comestibles del Perú. Pan del Futuro. Universidad de San Martín de Porres. Lima, Perú.

- Oliveira, E.C., Paula, E.J., Plastino, E.M., \& Petti, R. (1995). Metodologías para el cultivo no axénico de macroalgas marinas in vitro. En Alveal, K.; Ferrario, M. E.; Oliveira, E. C.; Sar, E. 1995. Manual de Métodos Ficológicos. Pág.: 429-447. Universidad de Concepción. Chile.

- Pacheco-Ruíz, I., Zertuche-González, J.A., Arroyo-Ortega, E., \& Valenzuela-Espinoza, E. (2004). Agricultural fertilizers as alternative culture media for biomass production of Chondracanthus squarrulosus (Rhodophyta, Gigartinales) under semi-controlled conditions. Aquaculture, 240(1-4):201-209.

- Piña, P., Medina, M.A., Nieves, M., Leal, S., López-Elías, J.A., \& Guerrero, M.A. (2011). Cultivo de cuatro especies de microalgas con diferentes fertilizantes utilizados en acuicultura. Revista de Investigaciones Marinas, 28(3): 225236.

- Redmond, S., Green, L., Yarish, C. H., Kim, J., \& Neefus, C. (2014). New England, Seaweed culture handbook. Nursery systems. University of Connecticut \& University of New Hampshire.

- Romo, H., Ávila, M., Candía, M., Nuñez, M., Oyarzo, C., Gallegillos, F., \& Cáceres, J. (2005). Manual de técnicas de cultivo de Luche (Porphyra sp.). Proyecto FONDEF D01 I 1148 IFOP, 32 pp.

- Sahoo, D., Tang, X., \& Yarish, C. (2002). Porphyra - the economic seaweed as a new experimental system. Current Science, 83(11):1313-1316. 
- Sahoo, D., \& Yarish, C. (2005). Mariculture of seaweeds. In: Andersen RA. eds. Algal Culturing Techniques. USA, Elsevier Academic Press. p. 219-237

- Salinas, J.M., \& Valdés, L. (1993). Influence of temperature and photoperiod on the reattachment process of Gelidium sesquipedale (Clem.) Born. Et Thur. (Gelidiales: Rhodophyta). Journal of Applied Phycology: 317-326.

- Shapiro, S.S., \& Francia, R.S. (1972). An approximate analysis of variance test for normality. Journal of the American Statistical Association, 67:215-216.

- Ugás, R. (2014). 40 verduras viejas y nuevas para diversificar tu alimentación y nutrirte mejor. Universidad Nacional Agraria La Molina. Lima, Perú.

- Valenzuela-Espinoza, E., Lafarga-De La Cruz, F., Millán-Núñez, R., \& Núñez-Cebrero, F. (2005). Crecimiento, consumo de nutrientes y composición proximal de Rhodomonas sp. cultivada con medio $\mathrm{f} / 2$ y fertilizantes agrícolas. Ciencias Marinas, 31(1A), 79-89.

- Werlinger, C., Mansilla, A., Villarroel, A., \& Palacios, M. (2008). Effects of photon flux density and agricultural fertilizers on the development of Sarcothalia crispata tetraspores (Rhodophyta, Gigartinales) from the Strait of Magellan, Chile. Journal of Applied Phycology, 20(5):757-765. 